\title{
A CASE OF GRANULOMA ANNULARE IN AN INFANT FOLLOWING MORBILLI-PAROTITIS-RUBELLA VACCINATION
}

\author{
Valentina Broshtilova ${ }^{1,2}$, Sonya Marina ${ }^{2}$ \\ ${ }^{1}$ Department of Dermatology and Venereology, Military Medical Academy, Sofia, \\ ${ }^{2}$ Department of Infectious Diseases, Parasitology and Dermatovenereology, \\ Faculty of Medicine, Medical University of Varna
}

\begin{abstract}
An 18-month-old female with granuloma annulare (GA), possibly related to morbilli-parotitis-rubella vaccination is reported. The first lesions started within ten days after injection and quickly disseminated over the distal aspects of the extremities. GA is relatively rare in this age group, and only anecdotal cases have been attributed to vaccination etiology. To the best of our knowledge, GA induced by morbilli-parotitisrubella vaccination has not been described before. Speculations on the mechanical or immunological GA
\end{abstract} triggers related to the vaccinations, together with a comprehensive review of literature, are highlighted.

Keywords: granuloma annulare, vaccination, morbilli-parotitis-rubel

First described in 1895 as localized annular eruption on the fingers of an adolescent, granuloma annulare (GA) is a benign, usually self-limiting inflammatory dermatosis of the dermis and subcutaneous fat (1), presenting with erythematous or skincolored papules, coalescent into ring-like plaques with predilection to the distal aspects of the extremities. Localized, generalized, disseminated, subcu-

\footnotetext{
Address for correspondence:

Valentina Broshtilova

Faculty of Medicine

Medical University of Varna

55 Marin Drinov St

9002 Varna

e-mail:broshtolova@mail.bg
}

Received: February 9, 2020

Accepted: March 24, 2020 taneous, and perforating clinical variants of GA are described.

We present an 18-month-old girl with eight erythematous ring-like plaques, symmetrically distributed over the dorsal aspects of the upper and lower extremities, which started to evolve two weeks after the routine morbilli-parotitis-rubella (MPR) vaccination done at the 13th month of age. No subjective symptoms, family history of atopy, specific hypersensitivity or metabolic disturbances were present. The physical examination revealed referential height, weight, and vital parameters. The clinical features were confirmed histologically to coin the diagnosis of granuloma annulare. Thyroid dysfunction, infectious etiology such as Borrelia burgdorferi, hepatitis $\mathrm{B}$ and $\mathrm{C}$, syphilis, and human immunodeficiency virus 1 and 2 were ruled out. The lesions showed a slow tendency of spontaneous resorption upon biopsy, which was additionally enhanced by topical ap- 
plication of mometasone furoate twice daily for two weeks, and disappeared at the first month follow-up.

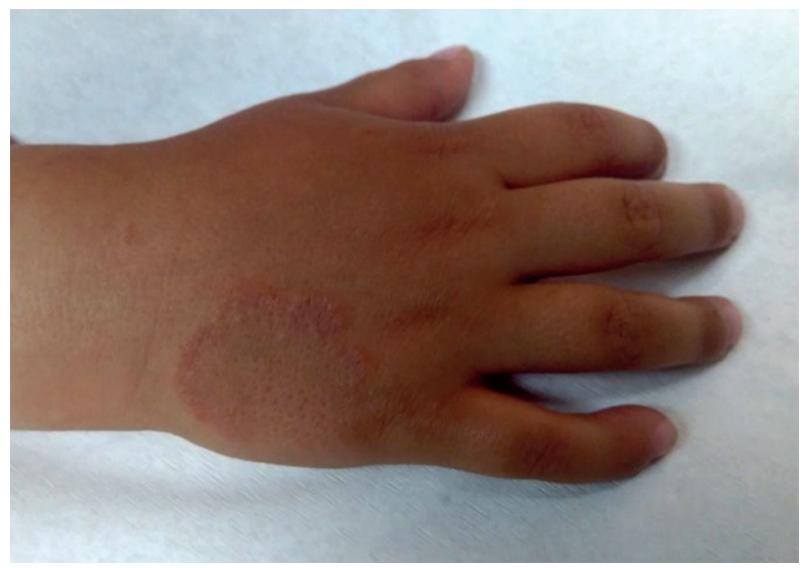

Fig. 1. Erythematous plaque with annular configuration on the dorsal hand

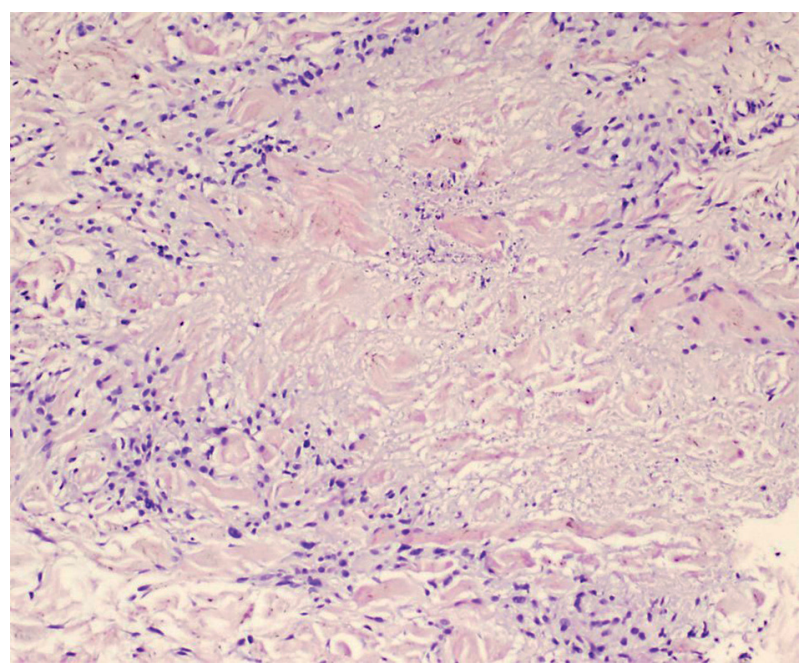

Fig. 2. Focus of degenerative collagen with mucin deposition surrounded by palisading granulomatous inflammation $(x$ 100, HE)

GA is a prototypic morphological phenomenon with versatile etiological profile. Typically, erythematous or flesh-colored papules, which confluent into annular plaques with central clearing and elevated borders, are present. The histological picture shows palisading granulomas with collagen degeneration and mucin deposit. The lesions are usually asymptomatic and symmetrically attached to distal parts of the extremities. Most often young adults are affected with twice more common female incidence. Infant cases are rare. To date, a total of 120 infants with GA have been described worldwide. Most cases are spo- radic and appear in healthy children. Familial predisposition has been related to HLA-associated subtypes such as HLA Bw 35 . There are twins, siblings and successive generations affected. The only congenital case reported dates back to 2005 (2).

The diagnosis relies on typical clinical findings. The most common clinical subforms in children are localized and subcutaneous. Generalized, linear, follicular pustular and perforatinghave been anecdotally described. The course is self-limiting with only short-term topical anti-inflammatory (corticosteroids or calcineurin inhibitors) treatment regimen sometimes applied. Surgical removal may be considered an appropriate therapeutic option for subcutaneous forms $(1,2)$.

The etiopathogenetic nature of the disease is extremely obscure. The main hypothesis suggests an auto-inflammatory immune response of delayed type, which may be triggered by minor mechanical trauma (especially in subcutaneous forms), glycerolization of the dermal-epidermal junction and collagen fibers in diabetes mellitus, thyroid dysfunction, and some hematological malignancies (myeloid leukemia and Hodgkin's disease) (1). The disseminated forms are attributed to excessive UV exposure. Infectious factors such as Borrelia burgdorferiare also considered.

A profound literature review reveals only 4 cases of vaccination-induced GA in children. Two followed a Bacillus Calmette-Guerin vaccination (3), one was induced by tetanus and diphtheria toxoid (4), and one was possibly triggered by antitetanus vaccination (5). The role of mechanical trauma on the site of injection could not be excluded either. The lack of other etiological factors and compatible delay supported the vaccine-induced pathogenesis in all reported cases. We describe a case of possible MPR-induced multiple localized GAin which the site of injection was not affected by the dermatological condition. Presumably, the vaccine initiated collagen necrobiosis through immunological mechanism.

Assembling the large number of vaccinations and the rather rare development of GA after vaccine injections, the possible inductive mechanism of vaccines in the etiopathogenesis of the disease requires more observations and a sophisticated scientific approach for further clarification. 


\section{REFERENCES}

1. Patrizi A, Gurioli C, Neri I. Childhood granuloma annulare: a review. G Ital Dermatol Venereol. 2014; 149(6):663-74.

2. De Aloe G, Risulo M, Sbano P, Pianigiani E, Fimiani M. Congenital subcutaneous granuloma annulare. Pediatr Dermatol. 2005; 22(3):234-6. doi: 10.1111/j.1525-1470.2005.22311.x.

3. Sang WL, Seung HG, Choi YW, Choi HY, Myung KB. Generalized granuloma annulare in infancy following Bacillus Calmette-Guerin vaccination. Ann Dermatol. 2011; 23(Suppl 3):319-22.doi: 10.5021/ad.2011.23.S3.S319.
4. Baskan EB, Tunali S, Kacar SD, Adim SB, Saricaoglu $\mathrm{H}$. A case of granuloma annulare in a child following tetanus and diphtheria toxoid vaccination. J Eur Acad Dermatol Venereol. 2005; 19(5):639-40. doi: 10.1111/j.1468-3083.2005.01206.x.

5. Baykal C, Ozkaya-Bayazit E, Kaymaz R. Granuloma annulare possibly triggered by antitetanus vaccination. J Eur Acad Dermatol Venereol. 2002; 16(5):516-8.doi: 10.1046/j.1468-3083.2002.00501.x. 